\title{
39 \\ Viral Infections of the Respiratory Tract
}

\author{
George L. Kirkpatrick
}

Viral infections of the respiratory tract are responsible for large amounts of time lost from the workplace and significant morbidity and mortality in the very young and the very old. The worldwide pandemic of influenza in 1918 was alone responsible for nearly 30 million deaths in excess of those expected for influenza. National Health Survey data for 1978 in the United States showed that acute respiratory illness accounted for $50 \%$ of all disabling conditions annually. ${ }^{1}$ That rate has continued unchanged for nearly 20 years. The common cold accounts for $40 \%$ of the respiratory illness and translates to more than 100 million disabling colds per year.

\section{Viruses Involved with Upper Respiratory Tract Infections}

Three studies, compared in Table 39.1, reflect prevalence rates for the most common respiratory tract viruses. A 12-month epidemiologic study of viral respiratory infections in Croatia from September 1, 1986 until August 31, 1987 involved 527 patients. $^{2}$ An Indian hospital-based study conducted from 1986 to 1989 on 736 children under the age of 5 years proved viral respiratory infections in $22 \%$ of the cases using nasopharyngeal cultures. ${ }^{3}$ Finally, in a setting of institutionalized elderly, Falsey and Treanor found 149 nursing home residents with upper respiratory tract illnesses during a 3-month period. ${ }^{4}$

Table 39.2 compares the relative frequency of each common respiratory virus in four studies. Dowling ${ }^{5}$ used epidemiologic data from numerous studies conducted during the late 1950s to define respiratory syndromes according to the viruses found by culture or rising antibody titers. Denny ${ }^{6}$ studied the relative role of various viral causes of acute respiratory infections in children enrolled in day-care. The 1987 data in North Carolina were similar to Dowling's pooled data from the 1950s. In South Africa from 1982 to 1991 McAnerney $^{7}$ collected 966 throat swab culture results from 16 viral watch centers. There were 4133 specimens received, with a positive culture result in $23.4 \%$ of cases. Monto and Sullivan ${ }^{8}$ studied families in Tecumseh, a small town in southeast Michigan, from 1965 to 1976 to determine the frequency of viral respiratory illnesses. Their results are similar to the others.

\section{Respiratory Syncytial Virus}

Respiratory syncytial virus (RSV), a single-stranded RNA paramyxovirus, is the leading cause of pneumonia and bronchiolitis in infants and children. Two antigenically distinct groups of RSV (A and B) are recognized. Community outbreaks of RSV usually appear during the winter and spring in temperate climates. The diagnosis of RSV in the acute setting is usually obtained by viral culture of nasopharyngeal secretions. A rapid diagnostic test (Abbott test pack RSV; Directigen RSV by Becton Dickinson) employing antigen detection in nasal secretions is $95 \%$ sensitive and $99 \%$ specific. Results are available in an hour.

The spectrum of illness associated with RSV is broad, ranging from mild nasal congestion to high fever and respiratory distress. What seems to begin as a simple cold may suddenly become a life-threatening illness. RSV tends to peak during early January most years. Evidence suggests that in infants group A viruses are associated with more severe infections than group B viruses. ${ }^{9}$ Modes of spread are primarily via large-droplet inoculation (requiring close contact) and self-inoculation via contaminated fomites or skin. RSV is recoverable from countertops for up to 6 hours from the time of contamination, from rubber gloves for up to 90 minutes, and from skin for up to 
Table 39.1. Prevalence of Common Upper Respiratory Tract Viruses

\begin{tabular}{|c|c|c|c|c|c|c|}
\hline \multirow[b]{2}{*}{ Virus } & \multicolumn{2}{|c|}{ Croatia study $^{2}$} & \multicolumn{2}{|c|}{ Indian Hospital study ${ }^{3}$} & \multicolumn{2}{|c|}{ Nursing home study ${ }^{4}$} \\
\hline & $\begin{array}{c}\text { Patients } \\
\text { (no.) }\end{array}$ & $\begin{array}{c}\text { Prevalence } \\
(\%)\end{array}$ & $\begin{array}{c}\text { Patients } \\
\text { (no.) }\end{array}$ & $\begin{array}{c}\text { Prevalence } \\
(\%)\end{array}$ & $\begin{array}{c}\text { Patients } \\
\text { (no.) }\end{array}$ & $\begin{array}{c}\text { Prevalence } \\
(\%)\end{array}$ \\
\hline Respiratory syncytial virus & 177 & 33.6 & 37 & 5.0 & 18 & 12.1 \\
\hline Rhinoviruses & & & & & 14 & 9.4 \\
\hline Herpes simplex & 8 & 1.5 & & & 6 & 4.0 \\
\hline Parainfluenza (types $1,2,3$ ) & 12 & 2.3 & 38 & 5.2 & 3 & 2.0 \\
\hline Influenza (types $\mathrm{A}$ and $\mathrm{B}$ ) & 3 & 0.6 & 45 & 6.1 & 0 & 0 \\
\hline Adenoviruses & 40 & 7.6 & 22 & 3.0 & & \\
\hline Enteroviruses & 18 & 3.4 & & & & \\
\hline Measles virus & & & 23 & 3.1 & & \\
\hline Mixed infections & 9 & 1.7 & & & 2 & 1.3 \\
\hline Total patients studied & 527 & - & 736 & - & 149 & - \\
\hline
\end{tabular}

Table 39.2. Frequency of Upper Respiratory Tract Viruses

\begin{tabular}{lccccc}
\hline & & \multicolumn{5}{c}{ Frequency (\%) } \\
\cline { 3 - 6 } Virus & Patients & Dowling $^{5}$ & Denny $^{6}$ & McAnernay & $\begin{array}{c}\text { Monto \& } \\
\text { Sullivan }^{8}\end{array}$ \\
\hline Adenovirus & 335 & 44 & 22 & 11 & 9 \\
Parainfluenza & 76 & 11 & 24 & 13 & 15 \\
Enterovirus & 101 & 14 & 14 & & 37 \\
Rhinovirus & 64 & 9 & 10 & & 22 \\
Influenza & 58 & 9 & 5 & 55 & 10 \\
Respiratory & 17 & 2 & 10 & 3 & \\
$\quad$ syncytial & & & & & \\
Coxsackie & 58 & 9 & 0 & & 93 \\
Corona & 20 & 2 & 0 & 82 & \\
$\quad$ Total & 729 & 100 & 85 & &
\end{tabular}

20 minutes. Viral shedding of RSV in infants is a prolonged process averaging 7 days.

Strategies for controlling spread of RSV should be aimed at interrupting hand carriage of the virus and selfinoculation of the eyes and nose. Masks commonly employed for respiratory viruses have not been shown to be an effective measure for curtailing RSV outbreaks on pediatric wards. Hand-washing is probably the single most important infection control measure for RSV.

\section{Influenza Viruses}

Influenza, often considered a benign disease today, has ravaged human populations recently enough that there are still those living who can recall the 1918 worldwide pandemic called the "Spanish flu." This particular influenza was described as beginning with what appeared to be an ordinary attack of influenza and rapidly developing into severe pneumonia. Two hours after admission the patients had mahogany-colored spots over the cheek bones, and within a few hours cyanosis began to spread over the face. Death shortly overcame them as they strug- gled for air and suffocated. Severe influenza pandemics occur about every 7 to 11 years. They are always associated with extensive morbidity and a marked increase in mortality.

During 1977-1978 the National Health Survey estimated that 101 million acute respiratory illnesses caused by all types of respiratory viruses were medically attended in the United States. As many as 20 million cases could have been prevented by effective influenza prophylaxis. In addition to the predominant influenza virus that invades an area each season, many types, subtypes, or variants are identified during each epidemic period. These antigenically distinct viruses produce "herald waves." For several successive years, a relatively small wave of infections with an antigenically distinct virus can occur during the second half of an epidemic and herald the epidemic virus for the following year. These "herald waves" are useful to epidemiologists for predicting the viral antigens that should be included in each season's vaccine.

During the early stage of an epidemic, a disproportionate number of cases involve school-age children, 10 to 19 
years old. Later in the epidemic, more cases are diagnosed in younger children and adults. The age shift suggests that the early spread of influenza viruses in a community is concentrated among schoolchildren.

Another characteristic of influenza virus is the decreased rate of infection in children living in urban areas compared to that of children in rural areas. In 1974 the rate of influenza B was four times greater for children living in rural areas of Michigan than in the urban areas. Children of low income families in urban areas tend to become infected earlier in life and have milder illnesses. When these children experience intensive exposure to influenza type B during their school years, they may have immunity that is relatively more protective than that of children in the rural areas, who had less prior exposure. ${ }^{10}$

\section{Parainfluenza Viruses}

Parainfluenza is a single-stranded RNA virus of which four serotypes and two subtypes are recognized (parainfluenza types 1, 2, 3, 4A, and 4B). Peak activity of parainfluenza illness tends to occur in the fall and spring. These viruses can cause acute bronchitis, pneumonia, and bronchiolitis in young children. Most persons have been infected with parainfluenza virus by age 5 . Immunity to parainfluenza is incomplete; and as with RSV, reinfection occurs throughout life. Parainfluenza types 1 and 2 tend to peak during the autumn of the year, whereas parainfluenza type 3 shows an increased prevalence during late spring. Adult infection results in mild upper respiratory tract symptoms, although pneumonia occasionally occurs. Outbreaks of parainfluenza types 1 and 3 have been reported from long-term care facilities. ${ }^{11}$ Illness is characterized by fever, sore throat, rhinorrhea, and cough. The rate of pneumonia is relatively high.

Most studies suggest direct person-to-person transmission. Parainfluenza is stable in small-particle aerosols at the low humidity found in hospitals. Outbreaks tend to proceed more slowly than influenza or other aerosol-spread infections. ${ }^{12}$ Infection control policies should emphasize handwashing and isolation of patients.

\section{Rhinoviruses}

Rhinovirus is the most frequent cause of upper respiratory tract infections in adults. More than 100 antigenic types have been identified, and reinfection occurs throughout life. In temperate climates, rhinovirus infection shows spring and fall peaks of activity. ${ }^{13}$ Rhinovirus infection is diagnosed by viral culture of nasopharyngeal secretions. In the healthy adult rhinovirus infection is self-limited and characterized by nasal congestion, rhinorrhea, and a mild sore throat. Although cough is common, direct viral invasion below the nasopharynx is rare. Cough is caused by reflex pathways. Unlike other respiratory viruses (influenza, adenoviruses) rhinovirus infections produce relatively minor damage to the nasal epithelium and probably no damage to the tracheal mucosa. Because rhinovirus replication is reduced at ele- vated body temperatures, direct invasion of the lower respiratory tract is unusual at all ages.

In one study, rhinovirus infections produced the first seasonal peak of respiratory infections in a long-term care facility. ${ }^{14}$ Seasonal peaks occur most frequently between September and November. Rhinoviruses are easily transmitted by contact with infected secretions. The most efficient modes of spread are hand-to-hand contact or direct contact with a contaminated surface followed by inoculation of the nose or conjunctiva. Rhinoviruses remain infectious for as long as 3 hours on nonporous surfaces. Transmission can be decreased by hand-washing and disinfection of environmental surfaces.

\section{Coronaviruses}

Coronaviruses are single-stranded RNA viruses that have been identified as a major cause of colds in the general population. Epidemics occur during the winter and early spring. ${ }^{15}$ Infections in volunteers have produced an illness similar to rhinovirus infection. Low grade fever was present in approximately $20 \%$ of the patients. Upper respiratory tract infection is the most common result of coronavirus invasion. Coronaviruses have also been associated with exacerbations of chronic pulmonary diseases.

Diagnosis is difficult because the organism is not easy to isolate, and serology is generally not available. In a study of 11 long-term care facilities by Nicholson and Baker, ${ }^{16}$ patients with respiratory illnesses were analyzed for evidence of coronavirus infections. Antibodies to the two most well studied antigenic strains of coronavirus (229 E and OC43) were detected by enzymelinked immunosorbent assay (ELISA). It was noted that illnesses were indistinguishable from RSV and influenza virus infections. Lower respiratory complications, such as pneumonia, occurred in one-fourth of the infected residents.

\section{Adenoviruses}

Adenoviruses are double-stranded DNA viruses, with 41 recognized serotypes that most commonly cause infections in children. Coryza, pharyngitis, pneumonia, pharyngoconjunctival fever, and epidemic keratoconjunctivitis are attributable to adenoviruses. Transmission can occur by aerosolized droplets, fomites, and hand carriage as well as the fecal-oral route. The virus can be isolated for prolonged periods from respiratory secretions, conjunctival secretions, and stools of infected patients. The identification of adenoviruses can be confirmed by viral cultures, but the genetic heterogeneity of adenoviruses makes the information of little value. Outbreaks are uncommon in long-term care facilities for children or elderly patients. Should an outbreak of adenovirus occur, strategies for control should take into account the various modes of transmission. 


\section{Other Viruses}

Herpes simplex and measles viruses are occasionally cultured from nasopharyngeal secretions of patients with upper respiratory infection. In one study ${ }^{3}$ measles virus was found in $3 \%$ of nasopharyngeal cultures. The study showed a case-fatality rate of $43 \%$ among patients with upper respiratory infection from whom the measles virus was cultured. Similarly, herpes simplex virus showed a prevalence of $1.3 \%$ in nasopharyngeal cultures. There was no speculation about its involvement as a causative agent in the upper respiratory symptomatology.

Coxsackie and echoviruses are small RNA picornaviruses of about 60 types and subtypes. Most are transmitted from human to human by the fecal-oral route or by large droplet spread. They tend to cause outbreaks during warm summer months. Clusters of cases are found in day-care centers, summer camps, and military camps. Coxsackie viruses are divided into two types, A and B, with about 20 subtypes of each. A variety of dissimilar syndromes vary with the age of the patient (Table 39.3).

\section{Disease Presentations}

\section{Common Cold}

The common cold, a disease of antiquity, is characterized by objective signs and subjective symptoms that are usually self-limited. Symptoms that occur with common colds include sneezing, watering of the eyes, nasal stuffiness, nasal obstruction, postnasal discharge, sore throat, hoarseness, cough, and sputum production. The common cold is a clinical diagnosis and lacks specificity because other ailments such as allergies and early symptoms of more serious illnesses mimic common cold symptoms. In the United States colds account for 23 million lost days of work and 26 million lost school days per year.

Table 39.3. Syndromes Caused by Coxsackie and ECHO Viruses, by Subtype

\begin{tabular}{lc}
\hline Syndromes & Subtypes \\
\hline Coxsackie type A viruses & $1-6,8,10,22$ \\
Herpangina & 21 \\
Common cold & 9 \\
Pneumonia & $5,10,16$ \\
Hand, foot, and mouth disease & \\
Coxsackie type B viruses & \\
$\quad$ Summer febrile illness with respiratory & All \\
$\quad$ symptoms & \\
Pleurodynia & All \\
Epidemic cervical myalgia & $1-5$ \\
ECHO viruses & \\
Summer febrile illness & \\
Common cold & All \\
\hline
\end{tabular}

\section{Diagnosis}

Rhinoviruses, coronaviruses, and RSV are most likely to cause cold symptoms. At times, influenza viruses, parainfluenza viruses, and adenoviruses do not cause their typical symptom patterns and appear as a minor illness. Five factors to consider in the differential diagnosis of the illness and virus are (1) age; (2) epidemiology; (3) physical findings; (4) progression of symptoms; and (5) laboratory tests.

Age. Rhinoviruses are by far the most common cause of respiratory illnesses in individuals age 5 to 40 years. Most of these infections manifest as the common cold. RSV and the parainfluenza viruses are common in those less than 2 years, manifesting as cold symptoms but suddenly progressing to more severe illness.

Epidemiology. Knowing the time of year and paying attention to the patterns of illness spreading through the community are helpful for distinguishing colds from other masqueraders.

Physical Findings. Physical findings should be limited to rhinorrhea and nasal congestion with minor contributions of other mild symptoms. Severe pharyngitis or any abnormal lung findings exclude the common cold from the diagnostic possibilities.

Progression of Symptoms. On the first day or two of other illnesses the prodromal symptoms may mimic a cold. After a few days, other illnesses progress to new or more severe symptoms, whereas a cold retains its original pattern. Patients should report changing symptom patterns to their physician.

Laboratory Testing. Influenza A and RSV can now be identified within 1 hour by rapid antigen tests available commercially. Nasopharyngeal washings are more likely to give a positive result than a nasal swab, but both are sensitive and specific. The other viruses may be identified in tissue cultures or by time-resolved fluoroimmunoassays (TRFIAs) that are less widely available.

\section{Management}

There are as many ways to manage the common cold as there are physicians and mothers. Antibiotics have no effect on the causative viruses. Antihistamines have been shown to be of little use because the kinin system rather than histamine is responsible for rhinorrhea and congestion. ${ }^{17-19}$ Antihistamines cause a generalized drying of the respiratory tract that may be unpleasant. More than 800 cold preparations are available over the counter in the United States..$^{20}$ The decongestant component in some remedies may be helpful but is dangerous for hypertensive patients. Aspirin, acetaminophen, and nonsteroidal antiinflammatory drugs (NSAIDs) are associated with a mild reduction in symptoms but have been shown to suppress the serum neutralizing antibody response and cause a highly significant increase in the rate of viral 
shedding. ${ }^{21,22}$ They should not be used routinely but, rather, targeted for reducing myalgias and malaise. Even fever reduction is not a sacred reason for use of acetaminophen and antiinflammatory agents. Elevated body temperature has been shown to protect puppies from fatal viral infection. ${ }^{23}$ Numerous studies have shown symptomatic improvement after raising the temperature of the nasal mucosa by inhaling heated vapor. ${ }^{24,25}$

Folk remedies such as hot chicken soup and vitamin C in large doses have large followings but no support in research literature. Zinc gluconate lozenges have enjoyed some interest as a possible treatment of common colds. Some research has shown strongly positive results with zinc. ${ }^{26}$ Doses large enough to give positive results usually cause unacceptable side effects. In the future, antirhinovirus drugs and interferon may reach development levels where alleviation of symptoms or prevention of infection exceeds side effects.

\section{Complications and Sequelae}

When the diagnosis of the common cold is accurate, complications and sequelae are minimal. Rhinoviruses and coronaviruses almost never progress to more serious disease. RSV, influenza virus, and parainfluenza virus infections frequently begin innocently but progress suddenly to life-threatening disease. Adenoviruses and coxsackie viruses may appear to cause a cold but frequently produce additional symptoms and follow a different course.

\section{Control and Prevention}

Hand-washing is the most important way to prevent transmission of these viruses. With rhinoviruses and RSV, direct contact with a contaminated surface followed by inoculation of the nose or conjunctiva can result in infection. Use of masks and gloves and isolation of infected persons is the most effective way to limit the spread of cold viruses. With minimal symptoms and multiple sources of infection in the community, however, these measures are not practical.

\section{Influenza}

Influenza has one of the more characteristic sets of clinical findings. The onset is usually sudden, with shivering, sweating, headache, aching in the orbits, and general malaise and misery. Cough is often found early in the course, aggravating headaches and causing generalized aching. The onset is generally explosive, with fever in adults ranging up to $102^{\circ} \mathrm{F}$. In children the fever may be higher than $102^{\circ} \mathrm{F}$, and sore throat may be an early sign. The most consistent signs are the presence of polymyalgias, weakness, and malaise.

\section{Diagnosis}

Not surprisingly, the diagnosis of influenza is more accurate during epidemics and less accurate during nonepidemic periods. Influenza in the United States usually occurs during December, January, and February. Successful presumptive diagnosis requires appropriate clinical symptomatology at the right time of the year and a knowledge of the pattern of influenzal illness around the world. Influenza affects all ages, but children under age 2 years and elderly patients may be more severely affected. The cluster of symptoms tends to remain unchanged from beginning to end except in babies and the elderly, in whom progression to lower respiratory involvement may herald life-threatening illness. When spread of an A type influenza virus is reported, the rapid flu A (Directigen; Becton Dickinson) test can confirm infection in less than an hour. This antigen-based test is $91 \%$ sensitive and $95 \%$ specific, requiring only a throat swab or nasopharyngeal washings for detection. Conventional viral cultures and complement fixation tests are used only for retrospective study because they take 4 to 8 weeks for results. No rapid test is yet available for B type influenza.

\section{Management}

Management of influenza is generally symptomatic. If the family physician has a strong suspicion that the virus in question is type A influenza, the patient may benefit greatly from the use of amantadine or rimantadine. Amantadine (Symmetrel) or rimantadine (Flumadine) in a dose of $200 \mathrm{mg} /$ day has long been known to be excellent prophylaxis against type A influenza. ${ }^{27}$ It is most effective if started early in the course of the disease, although it does reduce symptoms and shorten the course of illness even if started 3 to 5 days into the disease process. The therapeutic dose of amantadine or rimantadine is $100 \mathrm{mg}$ three times a day except in patients who have compromised renal function, for whom dosage adjustment is necessary. Because of the severity of the myalgias and headache associated with influenza, aspirin and NSAIDs may not suffice to relieve pain, and a narcotic-containing product is frequently indicated. With good instructions, the patient can receive double benefit from codeine or hydrocodone, which reduces not only the myalgias but the cough.

\section{Complications and Sequelae}

Pneumonia is usually blamed for excess mortality, but twice as many deaths during an influenza epidemic are attributable to ischemic heart disease than to pneumonia. ${ }^{28}$ From July 1, 1975 through June 30, 1976 Glezen $^{28}$ studied 3301 hospitalized patients with the admission diagnosis of acute respiratory disease. Influenza and other respiratory disease were the cause $21.4 \%$ of the time. Glezen also noted that the rate of hospitalization with acute respiratory disease during the influenza A/Victoria epidemic varied by age group. Infants under age 1 had a hospitalization rate of 160 per 10,000 patients. Patients between age 1 and 65 had low hospitalization rates, whereas those over age 65 had a rate of 167 per 10,000. The rates of hospitalization were low in the school-age group, which would have had the highest rates with other infections. 


\section{Control and Prevention}

Influenza vaccine is produced annually on the recommendation of the U.S. Food and Drug Administration (FDA) Vaccines and Related Biologicals Advisory Committee. Antigenic choices are based on (1) the viruses that have been seen during the previous year, (2) the viruses that are being seen in other parts of the world during the current year, and (3) the estimated antibody response in persons previously infected or vaccinated to these viruses.

There are two basic strategies for the use of vaccines and chemotherapy. A current strategy is to immunize high risk groups (the elderly and children with underlying conditions including heart, pulmonary, malignant, and some metabolic diseases). ${ }^{29}$ Healthy persons who become infected spread their infection to nonimmunized high risk children. Another approach to the control of influenza is to immunize all schoolchildren, children in day-care, college students, military personnel, and employees of large companies. These groups have the highest susceptibility and, because of the nature of their activities, are the principal vectors of influenza virus in the community.

\section{Bronchitis}

\section{Diagnosis}

Bronchitis is an inflammation of the major and minor bronchial branches. It is characterized by a cough that is frequently productive of sputum, depending on the inflammatory cause. Bacterial causes of bronchitis generally produce purulent-looking sputum. Viral causes of bronchitis can cause purulent-appearing sputum but more commonly produce either clear sputum or a nonproductive cough. On physical examination a patient with bronchitis has a noticeable cough, but the lungs are usually normal to auscultation except for a few scattered rhonchi. Rales, dullness to percussion, egophony, and other lower respiratory findings are usually absent. Cigarette smoking, other air pollutants, and chemical exposures that cause bronchial irritation may prolong an episode of bronchitis. Systemic lupus erythematosus is a cause of persistent bronchitis in a small number of affected patients. ${ }^{30}$

\section{Spectrum of Infection}

Studies indicate that viral causes of acute bronchitis tend to be more common with influenza (types A and B), parainfluenza of all four serotypes, and RSV. Coronavirus and adenoviruses cause bronchitis less commonly. RSV and parainfluenza viruses are found more commonly in the young population, and coronaviruses and adenoviruses occur in older patients. Influenza causes bronchitis at all ages. Rhinovirus is the most frequent cause of upper respiratory tract infection in adults and can be a cause of coughing without bronchitis.
Glezen, ${ }^{28}$ in a study of hospitalized patients with acute respiratory diagnoses, found acute bronchitis in $18.9 \%$. $\mathrm{He}$ also found that adult admissions for bronchitis showed an appreciable increase only during the influenza A/Victoria epidemic, and pediatric admissions for bronchitis increased during the autumn parainfluenza types 1 and 2 epidemic and the RSV outbreak in December. Falsey and Treanor ${ }^{4}$ reported that in institutionalized elderly patients RSV was likely to cause bronchitis, whereas rhinovirus was more likely to cause rhinorrhea with cough as a secondary symptom. Sputum production was considerably less frequent with the rhinovirus infection than with RSV infection.

\section{Spasmodic Croup and Laryngotracheobronchitis}

Spasmodic croup and laryngotracheobronchitis (LTB), or nonrecurrent croup, are different diseases caused by the same organisms (see Chapter 18). The focal points of their illnesses are croupy cough and inspiratory stridor. Spasmodic croup bursts into a family's life with a scary suddenness when their under-3-year-old child exhibits a croupy cough and stridor and appears dyspneic. This child has not been ill and has no fever. After a few frantic hours the croup clears, and the child is well until the next episode.

In contrast, LTB begins as a cold. For 3 to 5 days the young child has low grade fever, rhinorrhea, congestion, sore throat, malaise, and cough. Fever gradually rises to $104^{\circ}$ to $105^{\circ} \mathrm{F}$, and the seal-like cough worsens to the point of inspiratory stridor. This child may be hoarse and may be wheezing too. Rarely airway obstruction requires intubation or bronchoscopy.

Both croups are the result of infection by a group of viruses including RSV, parainfluenza, influenza, adenoviruses, and coronaviruses. Influenza and RSV are more common during the winter months. The others are found in a fall and spring pattern. These viruses are transmitted by hand-to-hand contact or large-droplet aerosols. Knowing that influenza type A or RSV is in the community may be useful, as specific treatments are available.

Patients with spasmodic croup usually respond to breathing cool air (or in some cases warm mist in a steamed bathroom) and require little additional therapy. Resistant cases of spasmodic croup and most LTB cases respond to racemic epinephrine $(0.05 \mathrm{ml} / \mathrm{kg}$ of $2.25 \%$ solution diluted in $3 \mathrm{ml}$ of saline per application) administered as an aerosol. More severe cases of LTB require hospitalization for hydration and oxygen administration. Occasionally airway obstruction requires intubation. A single dose of intravenous steroid may preclude the need for intubation.

\section{Bronchiolitis}

\section{Clinical Picture}

Bronchiolitis is an acute viral respiratory disease generally found in children less than 2 years old (see Chap- 
ter 18). The typical clinical presentation is an upper respiratory infection with cough that progresses to a more severe cough and tachypnea. Respirations become rapid and shallow with a prolonged expiratory phase. Because the infants are not able to breathe well, they are also unable to suck or drink and can become dehydrated.

\section{Diagnosis}

Physical findings include intercostal retractions and nasal flaring, which suggest pneumonia. A chest roentgenogram shows only hyperinflation with no infiltrates. Tight respiratory sounds (not entirely typical of wheezes found with asthma) are usually present, as are some rhonchi. Rales and dullness to percussion suggest the coexistence of pneumonia.

Bronchiolitis is most commonly caused by RSV, occurring predominantly during the winter and spring. Parainfluenza viruses, particularly types 1 and 2, can cause bronchiolitis during early winter. The most severe cases of bronchiolitis are usually caused by influenza viruses, especially type A. The virus involved can be identified by culture of nasopharyngeal secretions or rapid antigen tests for RSV and influenza A.

\section{Management}

Management of bronchiolitis depends on the progression of signs and symptoms. Hospitalization may be necessary to correct hypoxemia or dehydration. If fever is significant, pneumonia must be ruled out. Cases that appear to be recurrent bronchiolitis may be asthma, even if the child is less than 1 year old.

Outpatient treatment is generally supportive, with careful attention to hydration. If hospitalization becomes necessary to correct hypoxemia or dehydration, treatment is focused on oxygenation, mist, and mechanically clearing the upper airway.

\section{Complications and Sequelae}

The most serious complication of bronchiolitis is respiratory failure requiring ventilatory assistance. It is best managed with continuous positive airway pressure and oxygen. Steroids and antibiotics are of questionable benefit for treating even the most severe cases of bronchiolitis. Ribavirin (Virazole) is useful as a continuous aerosol when RSV causes bronchiolitis. Ribavirin $(6 \mathrm{~g}$ in $300 \mathrm{ml}$ of water) is aerosolized in a small croup tent 16 to 20 hours a day for a minimum of 3 days up to 6 days.

\section{Family Issues}

Family support is particularly important when infants require hospitalization. Many children subsequently wheeze with other viral respiratory illnesses, which alarms the family. The family needs to know that the long-term prognosis is excellent, although some research suggests that pulmonary function remains impaired in these children, especially those who have serious underlying respiratory or cardiovascular disease.

\section{Pharyngoconjunctival Fever}

Pharyngoconjunctival fever is an upper respiratory illness that affects teenagers and adults. It manifests as pharyngitis, cough, fever, headache, myalgias, malaise, and particularly conjunctivitis. This syndrome is caused by adenovirus serotypes 3 and 7, which are frequently found in natural bodies of water, reservoirs, and nonchlorinated swimming pools.

Symptoms may be similar to those of influenza. Conjunctivitis is generally not present with influenza but is always found with pharyngoconjunctival fever and usually at an early stage. There is a spring and summer seasonal prevalence. It can be diagnosed by viral cultures of nasopharyngeal and throat swabs or paired complement fixation antibody studies for adenovirus.

Management of pharyngoconjunctival fever is symptomatic. There is no indication for systemic antibiotic treatment or ophthalmic antibiotics. There are no longterm complications or sequelae. Recovery is generally within 1 to 2 weeks.

\section{Laryngitis}

There are six distinct causes of laryngitis, the most common being viral infections of the upper respiratory tract. Vocal cord tumors, allergies, and strain of the vocal cords caused by long periods of loud talking produce laryngitis. A fairly frequent cause of laryngitis is hard coughing associated with an upper or lower respiratory tract infection. The least frequent cause is a bacterial infection of the throat.

Most of the causes of laryngitis are obvious. ${ }^{30}$ Viral laryngitis is difficult to distinguish from the less frequent bacterial laryngitis, which might require antibiotic treatment.

Children over age 2 and adults rarely have significant swelling of the throat that would put them at risk of airway obstruction. Children under age 2 are more likely to develop airway obstruction. Viral causes of laryngitis include the parainfluenza viruses, rhinoviruses, adenoviruses, and influenza viruses.

Voice rest makes the greatest impact on recovery. $\mathrm{Pa}-$ tients who are able to gargle with warm, weak saline solution find it soothing. Patients should be told that laryngitis is not a serious disease, and that adequate time to recover is the only therapy in most cases.

\section{Herpangina}

Herpangina accounts for up to $5 \%$ of all pharyngitis during the spring and summer. Several coxsackie A subtypes are responsible for this common ailment of children and teenagers (Table 39.3). It is characterized by small numbers of vesicles on red bases that ulcerate on the soft palate and tonsillar pillars. The severity of the pain is out of proportion to the number and appearance of the small ulcers. Fever and headache are common. The punchedout red-based ulcers are so diagnostic that no cultures or 
Table 39.4. Patterns of Viral Illness in Children and Elderly Patients

\begin{tabular}{|c|c|c|c|}
\hline \multirow[b]{2}{*}{ Virus } & \multicolumn{3}{|c|}{ Signs and symptoms } \\
\hline & Young children & Adults & Elderly \\
\hline $\begin{array}{l}\text { Respiratory } \\
\text { syncytial virus }\end{array}$ & $\begin{array}{r}\text { Wheezing, bronchiolitis, } \\
\text { pneumonia, bronchitis }\end{array}$ & Nasal congestion and cough & $\begin{array}{l}\text { Nasal congestion, cough, } \\
\text { fever, pneumonia, } \\
\text { wheezing, bronchitis }\end{array}$ \\
\hline Influenza & $\begin{array}{l}\text { Sore throat, high fever, } \\
\text { myalgias, bronchitis, croup, } \\
\text { bronchiolitis, rhinorrhea, otitis } \\
\text { media }\end{array}$ & $\begin{array}{l}\text { Fever, headache, myalgias, } \\
\text { malaise, cough, weakness, } \\
\text { bronchitis, laryngitis }\end{array}$ & $\begin{array}{l}\text { Bronchitis, low grade fever, } \\
\text { sore throat, pneumonia }\end{array}$ \\
\hline Parainfluenza & $\begin{array}{l}\text { Croup, bronchitis, pneumonia, } \\
\text { sore throat, bronchiolitis }\end{array}$ & Common cold, laryngitis & $\begin{array}{l}\text { Rhinorrhea, sore throat, cough, } \\
\text { pneumonia, fever }\end{array}$ \\
\hline Rhinoviruses & Sore throat, rhinorrhea & $\begin{array}{l}\text { Rhinorrhea, sneezing, cough, } \\
\text { sore throat, laryngitis }\end{array}$ & Rhinorrhea, cough, sneezing \\
\hline Coronaviruses & Croup, sore throat & $\begin{array}{l}\text { Common cold, malaise, } \\
\text { headache, sore throat, low } \\
\text { grade fever }\end{array}$ & $\begin{array}{l}\text { Exacerbation of chronic } \\
\text { pulmonary disease, } \\
\text { pneumonia, bronchitis }\end{array}$ \\
\hline Adenoviruses & Croup, sore throat & $\begin{array}{l}\text { Coryza, sore throat, } \\
\text { pneumonia, pharyngo- } \\
\text { conjunctival fever, } \\
\text { keratoconjunctivitis, } \\
\text { laryngitis }\end{array}$ & Bronchitis rarely \\
\hline
\end{tabular}

prophylactic antibiotics are indicated. Treatment is symptomatic. Recovery is complete within 5 to 7 days.

\section{Viral Respiratory Tract Infections in Very Young and Very Old Patients}

Although most respiratory infections in children initially appear innocent, enough cases become severe to make them the third leading cause of death for infants 28 days to 1 year of age. Respiratory infections are the second leading cause of death from age 1 to 4 years.

Parents using the emergency room for evaluation of their children's colds have reasonable concern for the health of the child; but of 60,000 children seen at Cook County Hospital's Pediatric Emergency Room, Mayefsky and El-Shinaway ${ }^{31}$ found more than $50 \%$ of the parents needed only reassurance that their child was not seriously ill.

Respiratory syncytial, influenza, and parainfluenza viruses are responsible for severe disease with excess mortality in very young children and elderly patients, while producing mild to moderate disease in everyone else. See Table 39.4 for a comparison of the effects of each virus on the three age groups. Mullooly and Barker ${ }^{32}$ found rates of hospitalization three to five times higher during influenza epidemics for children under 4 years of age.
Many elderly persons living in long-term care facilities develop respiratory illnesses mistakenly attributed to bacterial pneumonia. Those illnesses due to RSV, influenza, and parainfluenza viruses frequently become severe and are responsible for excess mortality. A significant number of deaths during these infections are of cardiovascular origin rather than respiratory (see Chapter 24).

\section{References}

1. Acute conditions, incidence, and associated disability, United States, 1977-78. Washington, DC: US Department of Health Education and Welfare, 1977:3. PHS 79-1560.

2. Milinaric G. Epidemiological picture of respiratory viral infections in Croatia. Acta Med Iugosl 1991;45:203-11.

3. Jain A. An Indian hospital study of viral causes of acute respiratory infection in children. J Med Microbiol 1991;35: 219-23.

4. Falsey AR, Treanor JJ. Viral respiratory infections in the institutionalized elderly; clinical and epidemiology findings. J Am Geriatr Soc 1992;40:115-19.

5. Dowling HF. Clinical syndromes in adults caused by respiratory 1963;88:61-72. Viruses. Am Rev Respir Dis

6. Denny FW. Acute respiratory infections in children: etiology and epidemiology. Pediatr Rev 1987;9:5.

7. McAnerney JM. Surveillance of respiratory viruses. S Afr Med J 1994;84:473-7.

8. Monto AS, Sullivan KM. Acute respiratory illness in the community, frequency of illness and the agents involved. Epidemiol Infect 1993;110:145-60. 
9. McConnochie KM, Hall CB. Variation in severity of respiratory syncytial viruses with subtype. J Pediatr 1990;117:52-8.

10. Glezen WP. Consideration of the risk of influenza in children and indications for prophylaxis. Rev Infect Dis 1980;2:408-20.

11. Public Health Laboratory Service Communicable Disease Surveillance Center. Parainfluenza infections in the elderly 1976-1982. BMJ 1983;287:1619.

12. Graman PS, Hall CB. Epidemiology and control of nosocomial viral infections. Infect Dis Clin North Am 1989;3:815-41.

13. Gwaltney JM, Mandell GL, Douglas RG Jr. Principles and practices of infectious diseases. 3rd ed. New York: Churchill Livingstone, 1989:1399-1404.

14. Grose PA, Rodstein M. Epidemiology of acute respiratory illness during an influenza outbreak in a nursing home: a prospective study. Arch Intern Med 1988;148:559-61.

15. Larsen HE, Reed JE. Isolation of rhinoviruses and coronaviruses from 38 colds in adults. J Med Virol 1980;5:211-19.

16. Nicholson KG, Baker DJ. Acute upper respiratory tract illness and influenza immunizations in homes for the elderly. Epidemiol Infect 1990;105:609-18.

17. Berkowitz RB, Tinkelman DG. Evaluation of oral terfenadine for treatment of the common cold. Ann Allergy 1991; 67:593-7.

18. Gaffey JJ, Kaiser DL. Ineffectiveness of oral terfenadine in natural colds: evidence against histamine as a mediator of common cold symptoms. Pediatr Infect Dis J 1988;7: 215-42.

19. Hutton N, Wilson MH. Effectiveness of an antihistaminedecongestant combination for young children with the common cold: a randomized, controlled clinical trial. J Pediatr 1991;118:125-30.

20. Smith $\mathrm{MBH}$, Feldman W. Over the counter cold medications. JAMA 1993;269:2258-63.
21. Graham NMH, Burrell CJ. Adverse effects of aspirin acetaminophen and ibuprophen on immune function, viral shedding, and clinical status in rhinovirus-infected volunteers. J Infect Dis 1990;162:1277-82.

22. Stanley ED, Jackson GG. Increased virus shedding with aspirin treatment of rhinovirus infection. JAMA 1975;231: 1248-51.

23. Carmichael LE, Barnes FD. Temperature as a factor in resistance of young puppies to canine herpesvirus. J Infect Dis 1969;120:669-78.

24. Ophir D, Elad Y. Effects of steam inhalation on nasal patency and nasal symptoms in patients with the common cold. Am J Otolaryngol 1987;8:149-53.

25. Tyrrell D, Barrow I. Local hyperthermia benefits natural and experimental common colds. BMJ 1989;298:1280-3.

26. Eby GA, Davis DR. Reduction in duration of common colds by zinc gluconate lozenges in a double-blind study. Antimicrob Agents Chemother 1984;25:20-4.

27. Kobayashi JM. Control of influenza A outbreaks in nursing homes: amantadine as an adjunct to vaccine-Washington, 1989-90. MMWR 1991;40:841-4.

28. Glezen WP. Serious morbidity and mortality associated with influenza epidemics. Epidemiol Rev 1982;4:25-43.

29. Liou YS, Barbour SD, Bell LM. Children hospitalized with influenza B infection. Pediatr Infect Dis J 1987;6:541-3.

30. Raz E, Bursztyn M. Severe recurrent lupus laryngitis. Am J Med 1992;92:109-10.

31. Mayefsky JH, El-Shinaway Y. Families who seek care for the common cold in a pediatric emergency department. J Pediatr 1991;119:933-4.

32. Mullooly JP, Barker WH. Impact of type A influenza on children: a retrospective study. Am J Public Health 1982;72: 1008-16. 